\title{
An Illumination Estimation Algorithm based on Outdoor Scene Classification
}

\author{
Ning Li, Chunxiao Li, Songnan Chen, Jiangming Kan* \\ School of technology,Beijing Forestry University \\ No.35 Tsinghua East Road, Haidian District, Beijing, 10008. \\ China

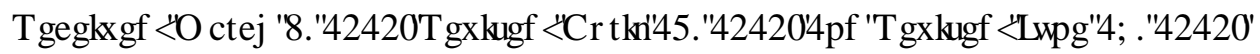

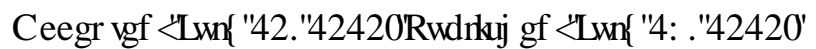

\begin{abstract}
The illumination estimation algorithm belongs to the field of color constancy, aiming to restoring the color of image through estimating the RGB of scene illumination. In different scenarios, the performance of a general algorithm varies greatly. If the scene can be predicted, it can be inferred that the scenarios related optimal algorithms is better than a general algorithm for estimating illumination. In this paper, a novel algorithm based on outdoor scene classification was proposed: firstly, the support vector machine (svm) classifiers was used to identify scene types, and then the scenarios related optimal algorithms was selected, finally used the RGB values of scene illumination were calculated.
\end{abstract}

Keywords - Illumination estimation, scene classification, SVM.

\section{INTRODUCTION}

$\mathrm{C}_{\mathrm{h}}$ olor constancy refers to the perceptual characteristics of human's perception. When the lighting of the scene changes, the light on the surface of the object in the scene changes accordingly, causing the color of the object to be distorted. Color constancy algorithm can restore image color, which mainly include color invariant extraction and illumination estimation algorithms. For computer vision, it is significant to achieve color constancy to restore the color of image. The color invariant algorithm restores the image color by extracting color invariants that are robust to illumination in the image; Theillumination estimation algorithm first estimates the R, G and B vector of lighting in the scene, and then performs matrix transformation according to the diagonal model to restore the color information.

Diagonal models have a long history in the field of color constancy. It was proposed by Kries, called the Von Kries diagonal model or diagonal model for short. Let the images obtained under two different lighting be I1 and I2 respectively, the elements in the diagonal matrix $\mathrm{M}$ are the estimated RGB values under illumination environment where I2 is located, then the diagonal model can be expressed as $I 1=M \cdot I 2$. For the calculation of color constancy, although the diagonal model is indispensable, it still has limitations and needs to be improved. Many scholars have also conducted related research [1], [2]. Worthey and Brill proposed that the effectiveness of the diagonal model depends largely on the function of the vision system sensor [3]; Worthey also pointed out that the use of narrow-band lighting with similar effects to narrow-band sensors can enhance color stability in the Retinex algorithm [3]; Finlayson et al. proposed that the linear combination of sensors in the vision system can be used to improve the diagonal model [4]. In short, the diagonal model is a relatively simple and effective mapping method in the field of color constancy.

The illumination estimation algorithm is divided into three categories: pixel-based algorithm, learning-based algorithm and combination algorithm [5]. Pixel-based illumination algorithm gains the RGB values of scene illumination according to the characteristics of low-level pixels, which starts from the Retinex theory proposed by Land and McCann, drawing on the human visual physiological characteristics [6]. The theory holds that the subtle changes in color are partly caused by changes in the color of light in the shooting scene, and dramatic changes in colors are caused by changes in the spectral reflectance of the surface of the object being shot in the scene; Based on this theory, Land and McCann proposed the Gray World and White Patch algorithm [6]. The algorithm is generally based on assumptions. For example, the white patch algorithm is based on the assumption that the color value corresponding to the maximum reflectance of the surface of the object being photographed in the scene is colorless; The Gray-World algorithm is based on the assumption that the average reflection of the surface of the object being shot in the scene is colorless. The average value in the RGB channel of the image is used as the scene illumination of the image. Under the constraints of assumptions, the effectiveness of these algorithms is limited by the statistical characteristics of the image. For example, the white patch algorithm has better color recovery in images with larger RGB values, while the Gray Word algorithm can achieve a satisfactory color restoration effect in images with richer colors. In order to improve the applicability of the Gray World algorithm, Xu et.al proposed two optimization methods, one is the Gray World algorithm based on weighting, and the other is the Gray World algorithm based on image entropy. Based on the weighted Gray World algorithm, the image is first divided into several small image blocks, and then the average and standard deviation of the three channels of each image block R, G, B are calculated, and the standard deviation of each image block is weighted calculate the R, G and B values of the global illumination color of the image [7]. The 
algorithm is simple to calculate and runs fast, but due to the limitations of assumptions, it is impossible to achieve good color restoration effects for different types of images.

The learning-based illumination estimation algorithm first extracts the effective features in the image, and then learns the model between the image features and the reference scene lighting RGB to obtain a trained model, which is used to estimate the lighting RGB value on the new image, and finally restore the color. The method can achieve better estimation effect. In 1990, Forsyth [8] proposed a Gamut mapping-based illumination estimation method as the first learning-based lighting estimation algorithm. This method assumes that in the same scene, the number of colors observed under a given light source is limited; the algorithm contains the colors in the image in a closed, bounded convex set, and then finds the color gamut and the best mapping between the standard color gamuts to estimate the scene lighting color of the image. In 2004, Finlayson et al. [9] applied two-dimensional discretization to the illumination estimation algorithm based on color gamut mapping, and proposed the color by correlation algorithm. In 2010, Gijsenij et al. [10] introduced higher-order image structures into the gamut mapping framework, and proposed first and second color gamut mapping illumination estimation algorithms. Another representative learning-based illumination estimation method is the Bayesian theory-based, which was proposed by Brainard and Freeman [11]. This algorithm establishes a priori conditions for standard lighting of images, through Bayesian theory, estimate the RGB value of the scene lighting color of the input image. In 2006, Brainard et al. [12] established a quantitative model of human color constancy, which included the relationship between psychophysical data and the estimated lighting obtained by Bayesian algorithm.

In recent years, some model-based illumination estimation algorithms have been proposed. For example, learning the linear relationship between $\mathrm{R}$ and $\mathrm{B}$ component of image reference scene illumination color, Banic and Loncaric proposed Color Cat algorithm [13]; Bianco et al. introduced convolutional neural network into the field of illumination estimation to learning the regression relation between the images and their reference scene illumination color [14]; Barron and Tsai utilized fast Fourier transform and shifted the image into the frequency domain space to get the model to estimate the RGB values of scene illumination [15]. Although the algorithm can achieve a better image color restoration effect, the training of the model requires a lot of data support and requires a lot of time consumption. The most important thing is that determination of a suitable model requires a large amount of work.

Through the above discussion, it is not difficult to find that the illumination estimation algorithms based on the underlying pixels has the advantages of simple calculation and fast running speed, but the generalization is poor, and the modeling can achieve a good image color restoration effect. Some researchers have suggested that the two methods can be combined to explore the relationship between scene features and lighting estimation algorithms based on underlying pixels $\mathrm{d}$ for different scenes, and a fusion-based lighting estimation algorithm is proposed. The method generally uses the method based on the underlying pixels as the candidate algorithm, and selects the appropriate light estimation algorithm based on the image or statistical characteristics or fuses these algorithms [16].

The algorithm includes two types: unsupervised fusion lighting estimation method uses the RGB value of scene lighting obtained by fusion of a single algorithm to estimate the scene lighting color of the image, such as calculating the weighted average of the RGB value of the scene lighting obtained by a single algorithm; the supervised fusion lighting estimation algorithm uses the features of the image to guide the linear combination of a single algorithm. Gijsenij and Gevers [17] use the best algorithm of each image as a label, classify the features of the image with SVM, and then select the best algorithm for the input image according to the obtained classification model. Bianco and Ciocca [18] estimate the lighting by dividing the image into two categories (indoor and outdoor). They not only design different strategies to select the appropriate method for each category, but also consider the low-level attributes of the image and use these attributes to construct decision forest to determine the best method for new images[19]. Wu et al. [20] used texture-based matching strategies to find similar images and used local regression to combine candidate methods. Weijer et al. [21] select different lighting assumptions based on the high-level semantic features of the image to estimate the RGB value of the scene lighting color of the input image. The main problem of this algorithm lies in the choice of algorithm and the extraction of image features. If the selected algorithm has poor light estimation accuracy, even if the image uses the optimal algorithm, it will not achieve good results; and if the selected image features cannot classify different scenes well, then they cannot play a good guiding role in the choice of algorithm.

In response to the discussion of the above two issues and the conclusion in [17], for outdoor image sets, we first selected a richer and better lighting estimation algorithm as the candidate image set, and then extracted the Weibull coefficients and SIFT operators of the image as features. Weibull coefficients can represent the texture characteristics and edge information, and SIFT operators can reflect the local structure of the image. Finally, a fusion-based lighting estimation algorithm for outdoor scenes is proposed. Firstly, the scene was classified, and then the optimal illumination estimation algorithm of each kind of scene was used to calculate the RGB value of scene illumination according to the literature [22].

\section{ILLUMINANT ESTIMATION METHODS}

In this paper, we present a solution based on outdoor scene classification. it is mainly divided into four steps: (1) extract the Weibull parameters and SIFT operator of the image as features; (2) classify the scene of the image; (3) select nine lighting estimation algorithms to form the candidate algorithm set, evaluate the effect of the algorithm is in different scenarios, and select the optimal algorithm; (4) the images of 
different scenes use different optimal algorithms to estimate the RGB value of illumination. Each step will be discussed separately below.

\section{A. Scene feature extraction}

The algorithm proposed in this paper uses SIFT descriptor [23] and Weibull distribution coefficient [24] as scene features of image.

SIFT descriptor, as a local feature of the image, has the characteristics of stability, multiplicity and expansion, and remains unchanged in the changes of scale, brightness and perspective [23]. The DoG pyramid is constructed, then the feature points $p=\left\{p_{1}, p_{2}, \cdots, p_{n}\right\}$ are detected in different scales, and finally the gradient distribution of pixels in the feature point $p_{i}$ domain is used to determine the direction parameters of each feature point to form the position, scale, and direction information $\left(x_{i}, y_{i}, \sigma_{i}, \theta_{i}\right)$ corresponding to each feature point to form features descriptor. Each feature point can be described by 16 seed points, and each seed point $P_{i}$ can be divided into 8 directions of gradient information. Then a feature point has a 128-dimensional sift feature vector, namely $P_{i}=\left\{s_{1}, s_{2}, \cdots, s_{128}\right\}$. Obviously one image can extract $128 \times \mathrm{n} \quad$ dimension sift descriptor $\left\{s_{1}^{1}, s_{2}^{1}, \cdots, s_{128}^{1}, \cdots, s_{1}^{n}, \cdots s_{128}^{n}\right\}$.

Weibull distribution can be used to simulate the edge response distribution of image, which is one of the key features of scene recognition [24]. Weibull distribution extracts the texture and contrast of image through modeling the image's edge response, shown in (1), in which $\mathbf{X}$ is the edge response of Gaussian derivative filter in one color channel, $C$ is a normalization constant, $\beta$ is the scale parameter of distribution, $\gamma$ is the shape parameter. A larger $\beta$ indicates more contrast, and a larger $\gamma$ indicates a finer texture. The Weibull parameters computed in opposite intensity space according to (2), and then the Weibull parameter of an image is $\mathbf{W}=\left(\beta_{o_{1}}, \gamma_{o_{1}}, \beta_{o_{2}}, \gamma_{o_{2}}, \beta_{o_{3}}, \gamma_{o_{3}}\right)$.

$$
\begin{aligned}
& w(\mathbf{x})=C \exp \left(-\frac{1}{\gamma}\left|\frac{\mathbf{x}}{\beta}\right|^{\gamma}\right) \\
& \left\{\begin{array}{l}
O_{1}=\frac{R-G}{\sqrt{2}} \\
O_{2}=\frac{R+G-2 B}{\sqrt{6}} \\
O_{3}=\frac{R+G+B}{\sqrt{3}}
\end{array}\right.
\end{aligned}
$$

For the image named $I$, it's SIFT descriptor and Weibull parameters will be assembled to the feature vector
$\mathbf{F}=\left(\beta_{o 1}, \gamma_{o 1}, \beta_{o 2}, \gamma_{o 2}, \beta_{o 3}, \gamma_{o 3}, s_{1}^{1}, s_{2}^{1}, \cdots, s_{128}^{1}, \cdots, s_{1}^{n}, \cdots s_{128}^{n}\right)$ and be normalized. Then the SVM classifier [25] is applied to the feature vector to give the corresponding category. In this article the images was classified to two categories: City and Open Country.

\section{B. Scene classification}

From [17] we know that there is a correlation between the feature distribution of different scenes and the corresponding optimal algorithm, and the feature distribution of the same scene image are similar. After obtaining the features of the image, the support vector machine was used as the scene classifier to classify the image. SVM was first proposed by Cortes and Vapnik in 1995. It has showed many unique advantages in solving small samples, nonlinear and high-dimensional pattern recognition. The radial basis function (RBF) is used in the SVM classifier. This kernel function is one of the commonly used functions of linear inseparable SVM. Compared with other kernel functions, RBF can map a sample to a higher-dimensional space, usually defined as a monotonic function of the Euclidean distance between any point $x$ and a center $x_{\mathrm{c}}$ in the space, which can be written as $\mathrm{k}\left(\left\|x-x_{c}\right\|\right)$, its effect is often local, that is, the function takes a small value when $\mathrm{x}$ is far from the center $x_{\mathrm{c}}$.

We mainly conduct experiments in wild and urban scenes. Giving training set $D=\left\{\left(\mathbf{x}_{1}, y_{1}\right),\left(\mathbf{x}_{2}, y_{2}\right), \cdots,\left(\mathbf{x}_{m}, y_{m}\right)\right\}$, where $y_{i} \in\{-1,+1\}, \quad \mathbf{x}_{i}$ is the feature of the $\mathrm{i}$-th image, $y_{i}=-1$ is urban scenes and $y_{i}=+1$ is wild scenes. The image in the test set can be used to obtain the scene type according to the classifier, and the illumination color calculation algorithm can be used to calculate the illumination color of the image.

\section{Selection of illumination estimation algorithms}

In the previous study, the performances of different illumination estimation algorithms had been counted in different scenes [22]. But most are time consuming and require learning phrase. Pixel-based algorithms [26], by contrast, don't need learning and is short time-consuming, and Gamut Mapping [27], as a learning-based algorithm, whose learning phrase is completed beforehand, they could be selected to make up algorithm set. So the elements of the algorithm set are : Gray-Edge(GE), 1st Gray-Edge(1st GE), 2nd Gray-Edge(2nd GE), Gray World(GW), White Patch(WP), GAMUT pixel, GAMUT edge, GAMUT intersection.

1) Pixel-based algorithms

We choose the Gray-Edge(GE), 1st Gray-Edge(1st GE), 2nd Gray-Edge(2nd GE), Gray World(GW), White Patch(WP) algorithms in the calculation framework by Finlayson and Trezzi as pixel-based algorithms: 


$$
\left.\iint\left|\frac{\partial^{n} f_{c, \sigma}(x)}{\partial x^{n}}\right|^{\rho} d x\right)^{\frac{1}{\rho}}=k e_{c}^{n, \rho, \sigma}
$$

let $c=\{R, G, B\}, \rho$ is norm, $\sigma$ is Gaussian filter scale parameters $\mathrm{n}$ is derivative order, $\mathrm{k}$ is multiplicative scalar constraints. The derivative is defined as the convolution of the image and n-th order Gaussian filter:

$\frac{\partial^{s+t} f_{c, \sigma}}{\partial x^{s} y^{t}}=f_{c} * \frac{\partial^{s+t} G_{\sigma}}{\partial x^{s} \partial y^{t}}$

The formulas and parameters corresponding to each algorithm are shown in Table 1:

Table 1: The formulas and parameters corresponding to each algorithm

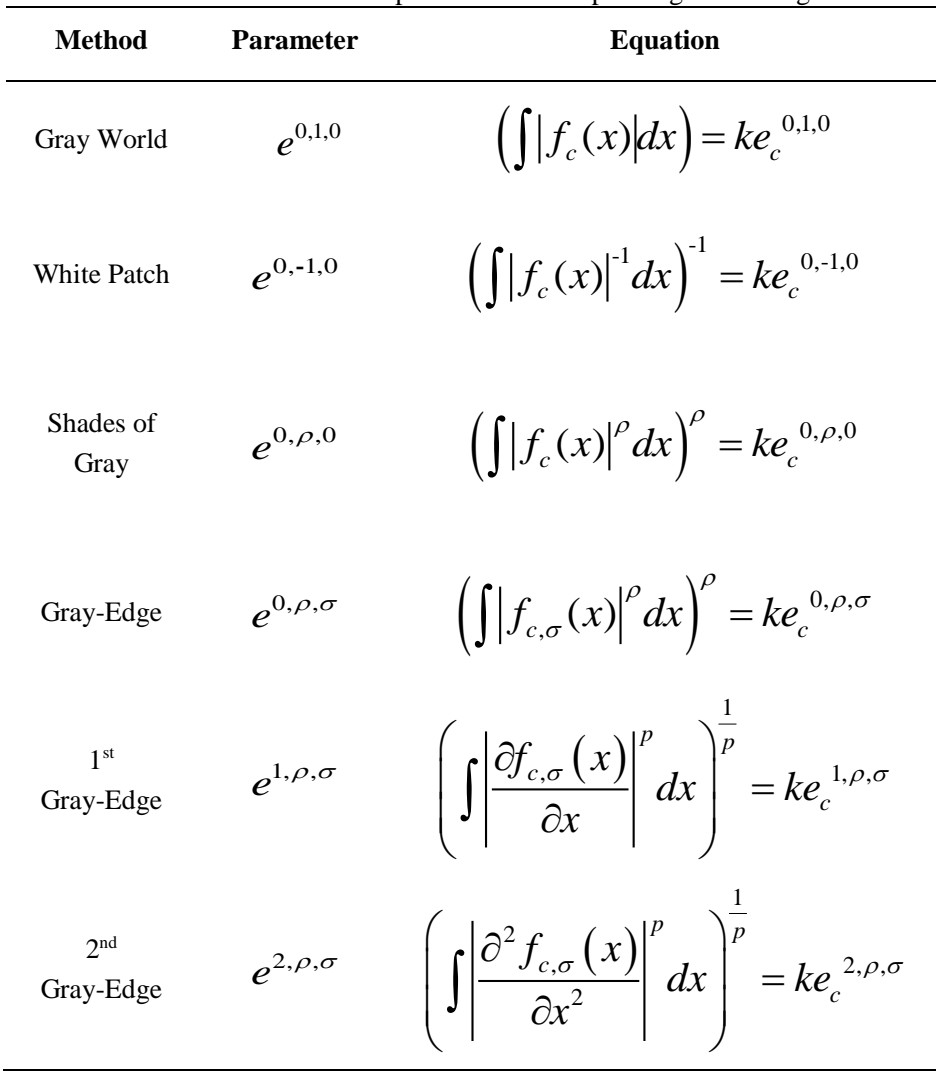

2) Gamut mapping

Gamut Mapping is one of the earliest learning-based lighting estimation algorithms and proposed by Forsyth in 1990. The algorithm is based on the assumption that in the real world, there are only a few color can be observed [8]. For example, if two colors are observed under a specific light source, then all colors between them can also be observed under that light source. Therefore, any change in the color of the image is caused by the deviation of the color of the light source. The classic color gamut is all colors observed by the observer under standard white light.

In general, the color gamut mapping algorithm takes an image get from an unknown light source and a pre-calculated standard color gamut as input, and estimates the illumination of the input image by matching the mapping between the color gamut in the input image and the standard color gamut color, the algorithm consists of three important steps, as shown in the Fig.1:

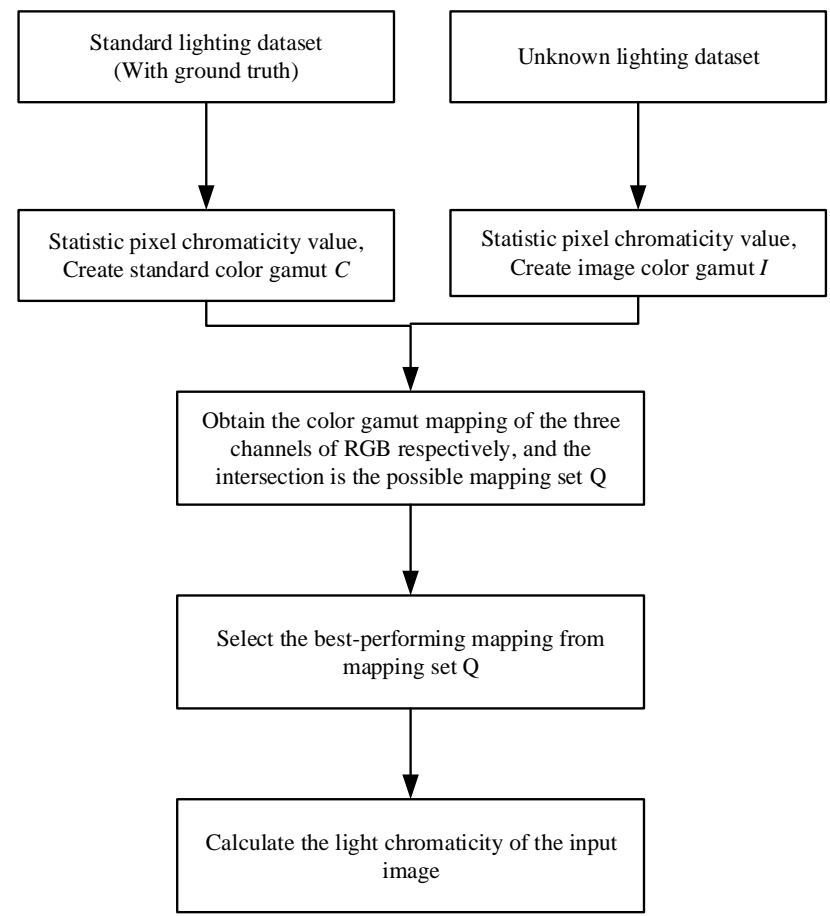

Fig. 1 Mapping steps between color gamuts

Gijsenij et al. proposed a color gamut mapping algorithm based on image differential structure, which uses the statistical characteristics of the differential structure of the image to calculate and obtain more image information. It can be expressed as:

$$
\left\{f, f_{x}, f_{y}, f_{x x}, f_{y y}, f_{x y}\right\}
$$

The differential of image $f$ is calculated by the convolution of image $f$ and Gaussian function $\mathrm{G}$.

$f \otimes \frac{\partial}{\partial x} G^{\sigma}=\frac{\partial}{\partial x}\left(f \otimes G^{\sigma}\right)$

The color gamut mapping algorithm based on the image differential structure is similar to the earliest proposed color gamut mapping algorithm. As the image order increases, the color gamut of the image becomes smaller and smaller, which can increase the possibility of selecting the correct mapping. Therefore, the mapping set of different differential structures of the image can be intersected or calculated to estimate the scene lighting color:

$$
\begin{aligned}
& \hat{M}_{\text {in }}=\bigcap_{i} M_{i} \\
& \hat{M}_{\text {un }}=\bigcup_{i} M_{i}
\end{aligned}
$$

We select GAMUT pixel, GAMUT edge, and GAMUT intersection in the gamut mapping algorithm to join the candidate algorithm set.

We consulted the literature [22] and compare the performance of eight algorithms on City scene and Open country scene in Table 2. As can be seen from Table 2, 
GAMUT pixel works best on Open country scene and White Patch performs best on City scene. So if the image is determined as Open country scene, use GAMUT pixel to estimate illumination; if the image is identified as City scene, use White Patch to estimate illumination.

Table 2 Error ranking of illumination estimation algorithms (based on the average value of reproduction angle error, unit: ${ }^{\circ}$ )

\begin{tabular}{|l|c|c|c|c|c|c|c|c|}
\hline \multirow{2}{*}{ Algonithms } & \multicolumn{4}{|c|}{ Open country } & \multicolumn{4}{c|}{ City } \\
\cline { 2 - 10 } & MEAN & MED & MAX & MIN & MEAN & MED & MAX & MIN \\
\hline GAMUT pixel & 6.86 & 6.12 & 27.16 & 0.05 & 6.91 & 6.25 & 24.94 & 0.05 \\
\hline GAMUT intersection & 7.28 & 6.55 & 28.45 & 0.06 & 4.17 & 3.14 & 19.79 & 0.05 \\
\hline GAMUT edge & 7.38 & 6.54 & 37.85 & 0.15 & 4.33 & 3.19 & 28.15 & 0.01 \\
\hline WP & 8.45 & 7.68 & 35.61 & 1.71 & 3.55 & 2.18 & 22.19 & 0.02 \\
\hline $2^{\text {sa GE }}$ & 8.61 & 7.79 & 34.13 & 0.32 & 3.68 & 2.32 & 20.18 & 0.05 \\
\hline 1" GE & 8.86 & 8.07 & 36.57 & 0.31 & 3.62 & 2.51 & 16.94 & 0.04 \\
\hline GE & 8.94 & 7.54 & 39.34 & 0.65 & 4.45 & 3.91 & 20.44 & 0.01 \\
\hline SOG & 8.94 & 7.54 & 39.34 & 0.65 & 4.45 & 3.91 & 20.44 & 0.01 \\
\hline GW & 9.83 & 8.58 & 40.29 & 0.5 & 5.69 & 4.88 & 20.84 & 0.13 \\
\hline
\end{tabular}

\section{EXPERIMENT}

\section{A. Image dataset}

Gray Ball dataset [28], Color Checker dataset [29] [30] are commonly used in the field of color constancy. Gray Ball dataset is proposed by SFU Laboratory. There are 11345 images totally and 13 scenes, including indoor and outdoor scenes. The RGB values of standard scene illumination for each image are captured by a gray sphere, which is mounted on the top of the camera. Gehler et al. used calibrated cameras to compose Color Checker dataset, which consists of 568 images, and is divided into indoor and outdoor scenes. All images contain a color checker, which can be used to get the RGB values of the scene illumination color of the corresponding image.

We selected the outdoor scene of Gray Ball dataset and Color Checker dataset as experimental dataset (EXP_data) to compare the performance of proposed algorithm with other out-of-state algorithms. EXP_data has 979 images, 574 of which are belonged to city scene and the other are belong to open country scene.

\section{B. Parameters selection}

In the process of SVM classification training, we used 3 -fold cross-validation to optimize the penalty parameter $\mathrm{c}$ and the parameter $\mathrm{g}$ of kernel function. The range of penalty parameter $\mathrm{c}$ and parameter $\mathrm{g}$ is set as. SVMcgForClass function in MATLAB is used to optimize these two parameters. This function utilizes the grid search method to optimize the parameters of the support vector machine. As shown in Fig. 2, the optimum value of $\mathrm{c}$ and $\mathrm{g}$ are 5.278 and 1.7411 respectively, and the optimal classification accuracy is $92.95 \%$

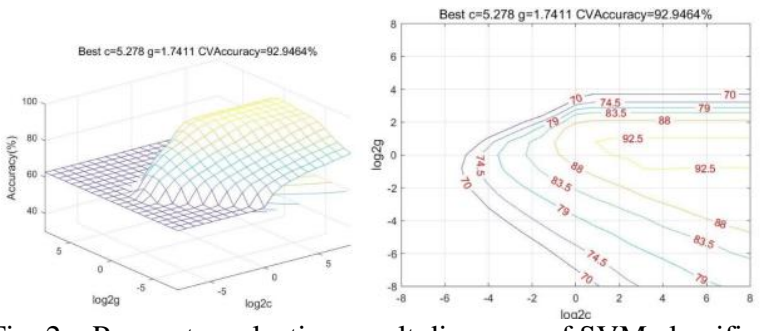

Fig. 2 Parameter selection result diagrams of SVM classification

\section{Performance evaluation}

We choosed angle error [31] and reproduction angle error [32] as algorithm performance evaluation.

Angle error is the angle deviation between the RGB of estimated illumination and standard illumination:

$$
E_{a}=\cos ^{-1}\left(\frac{\vec{e}_{a} \cdot \vec{e}_{e}}{\left\|\vec{e}_{a}\right\| \cdot\left\|\vec{e}_{e}\right\|}\right)
$$

Reproduction angle error is the angle between true white and estimated white (white surface under unknown light mapped to reference light using an illuminant estimate):

$$
E_{r e}=\cos ^{-1}\left(\frac{\vec{e}_{a} / \vec{e}_{e} \cdot U}{\left|\vec{e}_{a} / \vec{e}_{e}\right| \cdot \sqrt{3}}\right) \quad U \approx \frac{\vec{e}_{a}}{\vec{e}_{a}}
$$

We choosed mean, median, maximum, and minimum as the evaluation index. The smaller evaluation index means the estimated illumination color is close to the standard illumination color, and the corresponding algorithm is better

\section{Result analysis}

The proposed algorithm needs cross validation to make the result more effective. We used 5-fold cross-validation to determine the error of the algorithm on EXP_data. We compared our algorithm (C-C ILLU) with some illumination estimation algorithms: Gray-Edge (GE) [26], 1st Gray-Edge (1st GE) [26], 2nd Gray-Edge (2nd GE) [26], Gray World (GW) [33], White Patch (WP) [33], GAMUT pixel [29], GAMUT edge [29], GAMUT intersection [29], Exemplar-Based Color Constancy (EBCC) [34], Regression (SVR) [35], Bayesian[36], Color constancy using CNNs (CC_CNN) [27], Use Natural Image Statistics (UNIS) [37], Bottom-up+Top-down (BU+TD) [38], Bottom-up (BU) [38], Top-down (TD) [38].

Table 3. The error of compared algorithms on EXP_data

\begin{tabular}{|l|l|l|l|l|l|l|l|l|}
\hline \multirow{2}{*}{ methods } & \multicolumn{3}{|c|}{ ERROR1_REPRODUCTION $\left({ }^{\circ}\right)$} & \multicolumn{5}{|c|}{ ERROR2_ANGLE ${ }^{\circ}$ ) } \\
\cline { 2 - 9 } & $\begin{array}{l}\text { MEA } \\
\text { N }\end{array}$ & MED & MAX & MIN & MEAN & MED & MAX & MIN \\
\hline GW & 8.64 & 7.61 & 32.91 & 0.38 & 8.58 & 7.36 & 35.07 & 0.39 \\
\hline WP & 6.89 & 5.93 & 30.36 & 1.22 & 6.56 & 6.55 & 29.81 & 1.26 \\
\hline SOG & 7.60 & 6.59 & 32.05 & 0.41 & 7.33 & 7.36 & 31.55 & 0.37 \\
\hline GE & 7.58 & 6.58 & 32.03 & 0.41 & 7.34 & 7.37 & 31.54 & 0.38 \\
\hline $1^{\text {st }}$ GE & 7.24 & 6.41 & 29.12 & 0.22 & 6.82 & 6.86 & 28.79 & $\mathbf{0 . 2 0}$ \\
\hline $2^{\text {nd }}$ GE & 7.09 & 6.13 & 28.98 & 0.24 & 6.69 & 6.72 & 29.27 & 0.25 \\
\hline $\begin{array}{l}\text { GAMUT } \\
\text { pixel }\end{array}$ & 5.75 & 4.56 & 27.24 & 0.06 & 5.77 & 4.59 & 24.95 & 0.07 \\
\hline $\begin{array}{l}\text { GAMUT } \\
\text { edge }\end{array}$ & 6.22 & 5.15 & 37.96 & $\mathbf{0 . 0 2}$ & 6.17 & 5.15 & 26.34 & 0.02 \\
\hline $\begin{array}{l}\text { GAMUT } \\
\text { intersection }\end{array}$ & 6.10 & 5.16 & 28.53 & 0.07 & 6.15 & 5.22 & 25.95 & 0.07 \\
\hline EBCC & 7.02 & 5.40 & 37.43 & 0.10 & 7.46 & 5.63 & 35.60 & 0.09 \\
\hline
\end{tabular}




\begin{tabular}{|l|l|l|l|l|l|l|l|l|}
\hline SVR & 13.16 & 11.14 & 58.04 & 0.33 & 13.28 & 10.96 & 59.67 & 0.31 \\
\hline CC_CNN & 4.30 & 3.78 & $\mathbf{1 4 . 6 4}$ & 1.30 & 4.36 & 3.99 & $\mathbf{1 4 . 1 2}$ & 1.13 \\
\hline $\begin{array}{l}\text { Spatial } \\
\text { Correlations }\end{array}$ & 10.23 & 8.61 & 48.02 & 0.10 & 10.36 & 8.80 & 37.92 & 0.09 \\
\hline BU & 10.43 & 8.36 & 49.39 & 0.16 & 10.79 & 8.71 & 42.06 & 0.16 \\
\hline BU+TD & 9.73 & 7.31 & 49.42 & 0.16 & 10.07 & 7.50 & 42.11 & 0.16 \\
\hline TD & 10.09 & 8.18 & 56.19 & 0.09 & 10.39 & 8.48 & 43.83 & $\mathbf{0 . 0 2}$ \\
\hline UNIS & 9.73 & 7.04 & 43.67 & 0.08 & 9.90 & 7.27 & 40.37 & 0.07 \\
\hline C-C ILLU & $\mathbf{4 . 2 0}$ & $\mathbf{2 . 3 0}$ & 19.60 & 0.05 & $\mathbf{4 . 1 8}$ & $\mathbf{2 . 2 8}$ & 19.69 & 0.05 \\
\hline
\end{tabular}

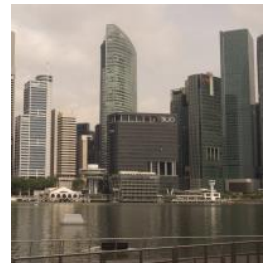

(1) input

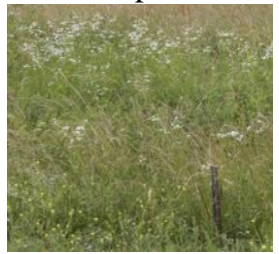

(1) input

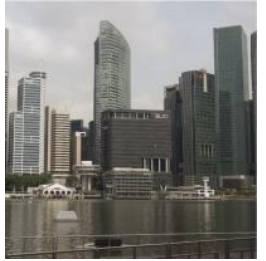

(2) proposed

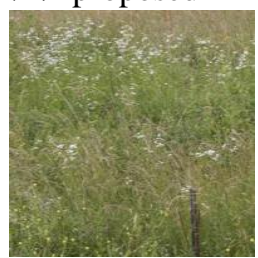

(2) proposed

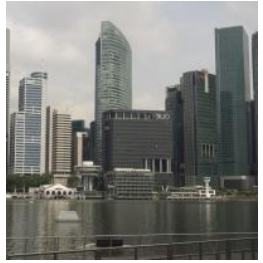

(3) groundtruth

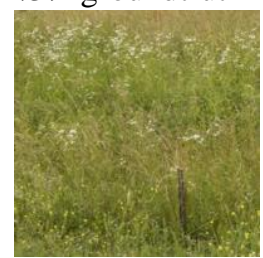

(3) groundtruth
Fig. 3 Experiment results

Table 3 shows the comparison between ours method and others in the EXP_data dataset, where the bold is the optimal value under each index.

The proposed algorithm (C-C ILLU) has an average error of 4.2 and a median error of 2.3. It is the smallest of all the comparison algorithms and has the best effect. Compared with the optimal algorithm WP for urban scenes and the optimal algorithm GAMUT pixel for wild scenes, the average value of the error is reduced by 2.69 and 1.55 , and the median value of the error is reduced by 3.63 and 2.26 respectively, which improves the two algorithms more. Taking the angle error as an indicator, we can see from Table 3 that the algorithm proposed in this paper (C-C ILLU) .The average error is 4.18 , and the median error is 2.28 . It is the smallest among all the comparison algorithms and has the best effect. Compared with the optimal algorithm WP for urban scenes and the optimal algorithm GAMUT pixel for wild scenes, the average error is reduced by 2.38 and 1.59 respectively. The median error is reduced by 4.27 and 1.59 respectively, and the effect of the algorithm is also greatly improved.

In summary, the effect of our proposed method is better. The color recovery effect is as shown in the above figure 3 . Compared with Ground True, it can be seen that the color recovery effect of ours is close to it, which can achieve a better overall color recovery of the image.

\section{CONCLUSION}

This paper presents an algorithm for outdoor scene lighting estimation. First the image features are extracted, the image is divided into two types: urban scene and outdoor scene, and then the optimal algorithm for each scene through experiments is obtained. Finally, the corresponding optimal algorithm estimates the illumination of the image, thereby color restoration is performed. The experimental results show that proposed algorithm is superior to other algorithms. In the future, more scenes and more illumination estimation algorithm are welcomed to be added to enrich the study of illumination estimation.

\section{ACKNOWLEDGEMENT}

This work was supported by National Natural Science Foundation of China (Grant No. 31570713) and Beijing municipal construction project special fund.

\section{References}

[1] West G, Brill M H. Necessary and sufficient conditions for Von Kries chromatic adaptation to give color constancy [J]. Journal of Mathematical Biology, 1982, 15(2):249-258.

[2] Worthey J A. Limitations of color constancy [J]. Journal of the Optical Society of America A, 1985, 2(2):1014-1026

[3] Worthey J A, Brill M H. Heuristic analysis of von Kries color constancy $[\mathrm{J}]$. Journal of the Optical Society of America A Optics \& Image Science, 1986, 3(10):1708-1712.

[4] Finlayson G D, Drew M S, Funt B V. Spectral sharpening: sensor transformations for improved color constancy $[\mathrm{J}]$. Journal of the Optical Society of America A, 1994, 11(5):1553-1563.

[5] Blackwell K T, Buchsbaum G. Quantitative studies of color constancy $[\mathrm{J}]$. Journal of the Optical Society of America A Optics \& Image Science, 1988, 5(10):1772.

[6] Land, Edwin H. The Retinex theory of color vision [J]. Scientific American, 1977, 237(6):108-128.

[7] Xu Xiaozhao, Cai Yiyan, Liu Xiaomin. Improved Grey World Color Correction Algorithms [J]. Acta Photonica Sinica, 2010, 39(3):559-564.

[8] Forsyth D A. A novel algorithm for color constancy [J]. International Journal of Computer Vision, 1990, 5(1):5-35.

[9] Finlayson G D, Trezzi E. Shades of gray and color constancy $[\mathrm{C}] / /$ Proceedings of The Twelfth Color Imaging Conference: IS\&T-The Society for Imaging Science and Technology, 2004:37-41.

[10] Gijsenij A, Gevers T, Weijer J V. Generalized Gamut Mapping using Image Derivative Structures for Color Constancy [J]. International Journal of Computer Vision, 2010, 86(23):127-139.

[11] Brainard D H, Freeman W T. Bayesian color constancy [J]. Journal of the Optical Society of America A Optics Image Science \& Vision, 1997, 14(7):1393.

[12] Brainard D H, Philippe Longère, Delahunt P B , et al. Bayesian model of human color constancy [J]. Journal of Vision, 2006, 6(11):1267-1281

[13] Banic N, Loncaric S. Color Cat: Remembering colors for illumination estimation [J]. IEEE Signal Processing Letters, 2015, 22(6):651-655.

[14] Bianco S, Cusano C, Schettini R. Color constancy using CNNs [C]//Proceedings of 2015 IEEE Conference on Computer Vision and Pattern Recognition Workshops (CVPRW): IEEE, 2015: 81-89.

[15] Barron J T, Tsai Y T. Fast fourier color 
constancy[C]//Proceedings of 2017 IEEE Conference on Computer Vision and Pattern Recognition: IEEE, 2017:6950-6958.

[16] Cardei V C, Funt B V. Committee-based color constancy $[\mathrm{C}] / /$ Proceedings of IS\&T/SID's Color Imaging Conference: IS\&T-The Society for Imaging Science and Technology, 1999:311-313.

[17] Gijsenij A, Gevers T, Weijer J V. Generalized Gamut Mapping using Image Derivative Structures for Color Constancy [J]. International Journal of Computer Vision, 2010, 86(23):127-139.

[18] Bianco S, Ciocca G, Cusano C , et al. Improving color constancy using indoor-outdoor image classification [J]. IEEE Transactions on Image Processing, 2008, 17(12):2381-2392.

[19] Bianco S, Ciocca G, Cusano C, et al. Automatic color constancy algorithm selection and combination [J]. Pattern Recognition, 2010,43 (3):695-705.

[20] Wu M, Sun J, Zhou J, et al. Color constancy based on texture pyramid matching and regularized local regression [J]. Journal of the Optical Society of America A, 2010, 27(10):2097-2105.

[21] Weijer J V D, Gevers T, Gijsenij A. Edge-based color constancy [J]. IEEE Transactions on Image Processing, 2007, 16(9):2207-2214

[22] Chunxiao L, Jiangming K. A performance evaluation of color constancy methods based on illumination estimation [J]. International Journal of Circuits, Systems and Signal Processing, 2018, 12: 35-41.

[23] Lowe D G. Distinctive image features from scale-invariant keypoints [J]. International Journal of Computer Vision, 2004, 60(2): 91-110.

[24] Geusebroek J M, Smeulders A W M. A six-stimulus theory for stochastic texture [J]. International Journal of Computer Vision, 2005, 62(1-2):7-16.

[25] Hsu C W, Lin C J. A Comparison of Methods for Multiclass Support Vector Machines [J]. IEEE Transactions on Neural Networks, 2002, 13(2):415-425.

[26] Weijer J V D, Gevers T, Gijsenij A. Edge-based color constancy $[\mathrm{J}]$. IEEE Transactions on Image Processing, 2007, 16(9):2207-2214.

[27] Gijsenij A, Gevers T, Weijer J V. Generalized Gamut Mapping using Image Derivative Structures for Color Constancy $[\mathrm{J}]$. International Journal of Computer Vision, 2010, 86(23):127-139.

[28] Ciurea F, Funt B. A large image database for color constancy research [C]//Proceedings of Color and Imaging Conference. 2003:160-164.

[29] Lilong, S., Brian, F. Re-processed version of the gehler color constancy dataset of 568 images, http://www.cs.sfu.ca/ colour/data/shi_gehler/.

[30] Peter, G., Carsten, R., Andrew, B., Tom, M., Toby, S. Bayesian Color Constancy Revisited [C]//Proceedings of IEEE Computer Society Conference on Computer Vision and Pattern Recognition: IEEE, 2008: 1063-6919.

[31] Barnard K, Cardei V, Funt B. A comparison of computational color constancy algorithms - Part I: Methodology and experiments with synthesized data [J]. IEEE Transactions on Image Processing, 2002, 11(9):972-983.
[32] Finlayson G D, Zakizadeh R , Gijsenij A . The reproduction angle error for evaluating the performance of illuminant estimation algorithms [J]. IEEE Transactions on Pattern Analysis and Machine Intelligence, 2017, 39(7):1482-1488.

[33] Finlayson G D, Trezzi E. Shades of gray and color constancy $[\mathrm{C}] / /$ Proceedings of The Twelfth Color Imaging Conference: IS\&T-The Society for Imaging Science and Technology, 2004:37-41.

[34] Joze H R V, Drew M S. Exemplar-Based Color Constancy and Multiple Illuminations $[\mathrm{J}]$. IEEE Transactions on Pattern Analysis and Machine Intelligence, 2014, 36(5):860-873.

[35] Wu M, Sun J, Zhou J, et al. Color constancy based on texture pyramid matching and regularized local regression [J]. Journal of the Optical Society of America A, 2010, 27(10):2097-2105.

[36] Brainard D H, Philippe Longère, Delahunt P B , et al. Bayesian model of human color constancy [J]. Journal of Vision, 2006, 6(11):1267-1281.

[37] Gijsenij A, Gevers T. Color constancy using natural image statistics and scene semantics [J]. IEEE Transactions on Pattern Analysis and Machine Intelligence, 2011, 33(4):687-698.

[38] Khan F S, Weijer J V D, Vanrell M. Top-down color attention for object recognition [C]//Proceedings of 2009 IEEE 12th International Conference ce, pp.160-164, 2003.

Ning Li, Phd in Engineering, director of experimental center, senior experimenter, main research areas iclude mechanical design and AI.

Chunxiao Li, Master Student in forestry engineering, main research areas include computer vision and Artificial intelligence.

Songnan Chen, PhD. Candidate in forestry engineering, main research areas include computer vision and Artificial intelligence.

Jiangming Kan, professor, Phd in Engineering, main research areas include forestry equipment and informatization and Computer Vision.

\section{Creative Commons Attribution License 4.0 (Attribution 4.0 International, CC BY 4.0)}

This article is published under the terms of the Creative Commons Attribution License 4.0

https://creativecommons.org/licenses/by/4.0/deed.en_US 